\title{
INVESTIGAÇÕES GEOFÍSICAS NA BORDA DA BACIA SEDIMENTAR DE SÃO PAULO
}

\author{
Welitom Rodrigues Borges, ${ }^{1}$ Jorge Luís Porsani² \\ Recebido em 19 jul., 2002 / Aceito em 13 dez., 2003 \\ Received Jul. 19, 2002 / Accepted Dec. 13, 2003
}

\section{ABSTRACT}

This paper shows partial results of geophysical investigations carried out in the border of São Paulo Sedimentary Basin, within the São Paulo University campus. The main objective of this research is characterizing the sediments and the top of basement rocks using the GPR - Ground Penetrating Radar and Resistivity Methods. The geophysical profiles were carried out along the same line in order to compare results obtained from these methodologies. One GPR profile (216m) was acquired employing $50 \mathrm{MHz}$ antennas and one resistivity profile with dipole length of $20 \mathrm{~m}$. Besides two verticals electrical soundings (VES) measurements were done on the same line. The analysis of GPR profile permitted to identify the bottom boundary the soil and one coarse sand layer. The result was supported by borehole information. The electrical profile identified one conductivity anomaly zone between 80 and 110m, related probably with a shear zone.

Keywords: GPR-Ground Penetrating Radar, Electrical Resistivity, São Paulo Sedimentary Basin, Campus of São Paulo University.

\section{RESUMO}

Este artigo mostra resultados parciais de investigações geofísicas realizadas na borda da Bacia Sedimentar de São Paulo, dentro do campusda Universidade de São Paulo. 0 principal objetivo desta pesquisa é a caracterização dos sedimentos e do topo das rochas do embasamento da bacia, utilizando os métodos GPR-Ground Penetrating Radar e Resistividade Elétrica. Os perfis geofísicos foram realizados ao longo de uma mesma linha, visando comparar os resultados com as duas metodologias. Um perfil de $216 \mathrm{~m}$ foi adquirido com as antenas de $50 \mathrm{MHz}$ e um perfil de resistividade com dipólos de $20 \mathrm{~m}$. Além disso, duas Sondagens Elétricas Verticais - SEV's foram feitas sobre a mesma linha. A análise do perfil GPR permitiu identificar o limite entre a base do solo e uma camada de areia. Os resultados estão de acordo com as informações de poços. 0 perfil elétrico identificou uma zona anômala de condutividade entre 80 e $110 \mathrm{~m}$, relacionada provavelmente com uma zona de cizalhamento.

Palavras-chave. GPR-Ground Penetrating Radar, Resistividade elétrica, Borda da Bacia Sedimentar de São Paulo.

\footnotetext{
Pós-Graduação em Geofísica-IAG/USP - Rua do Matão, 1226 - Cidade Universitária - São Paulo-SP - CEP: 05508-090 - Tel.: (11) 3091-2792 - Fax: (11) 3091-5034 - E-mail: welitom@iag.usp.br

2 IAG-USP - Departamento de Geofísica - Rua do Matão 1226 - Cidade Universitária - São Paulo - SP - CEP: 05508-090 - Tel.: (11) 30914734 - Fax: (11) 30915034 - E-mail: porsani@iag.usp.br
} 


\section{INTRODUÇÃOO}

Neste trabalho utilizaram-se os métodos GPR e eletrorresistividade para caracterizar os sedimentos e o topo rochoso granito-gnáissico na borda da Bacia Sedimentar de São Paulo. A área de pesquisa localiza-se em frente ao prédio do Instituto Astronômico e Geofísico (IAG/USP), situado na Rua do Matão, 1226, Campus da Universidade de São Paulo
(USP), porção centro-oeste do município de São Paulo, na margem esquerda do Rio Pinheiros (Figura 1).

\section{GEOLOGIA DA ÁREA DE ESTUDOS}

A Bacia Sedimentar de São Paulo está situada na porção central do conjunto de bacias tafrogênicas meso-cenozóicas do sudeste brasileiro.

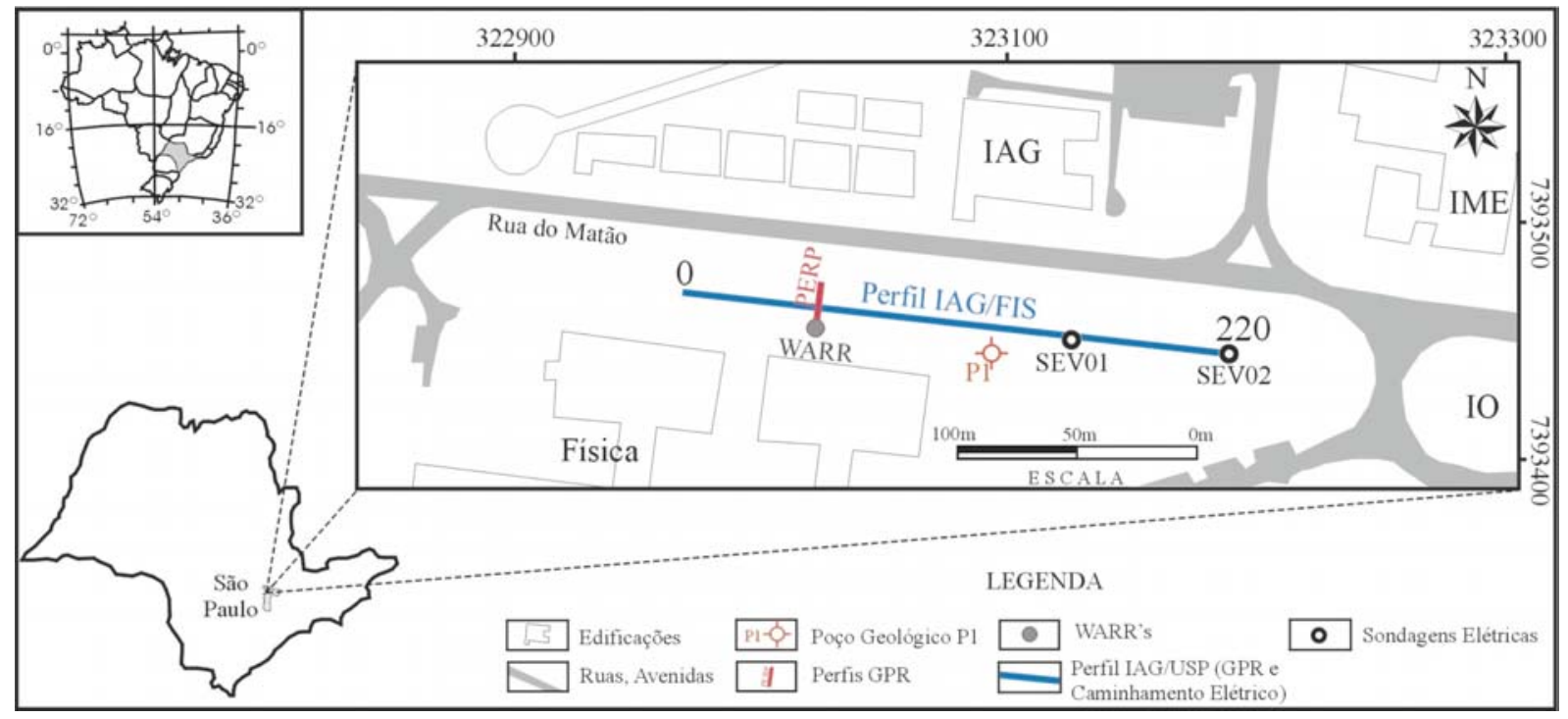

Figura 1 - Localização da área de pesquisa.

Figure 1- Location of research area.

Exibe forma irregular, aproximadamente elíptica, com uma área total de aproximadamente $1000 \mathrm{~km}^{2}$ e espessura máxima de $310 \mathrm{~m}$.

$\mathrm{Na}$ área em estudo predominam sedimentos areno-argilosos de idade terciária (formações São Paulo e Itaquaquecetuba), que capeiam o embasamento gnáissico de idade pré-cambriana, com diferentes graus de intemperização (IRITANI, 1993). Através da colaboração do projeto Investigações Geofísicas de Subsuperfície e de Poço na Borda da Bacia Sedimentar de São Paulo, financiado pela Fapesp, foi obtido um perfil litológico (Figura 2) de um poço executado em frente ao IAG, fundamental para as interpretações geológicas e geofísicas (PORSANI, 2001).

\section{METODOLOGIA E AQUISIÇÃO DOS DADOS}

0 GPR é um método eletromagnético que emprega ondas de rádio entre 10 e $1000 \mathrm{MHz}$, para localizar estruturas e feições geológicas rasas. A metodologia consiste em obter uma imagem de alta resolução através da emissão de um curto pulso de alta freqüência para gerar ondas eletromagnéticas que são radiadas para a subsuperfície por uma antena transmissora colocada na superfície. A propagação do sinal depende da frequência do sinal transmitido e das propriedades elétricas dos materiais, no caso, condutividade elétrica e permissividade dielétrica, que variam principalmente com o conteúdo de água no solo (PORSANI, 1999). A mudança nas propriedades elétricas em subsuperfície faz com que parte do sinal seja refletido. As ondas refletidas e difratadas em diferentes interfaces são recebidas por uma antena receptora colocada na superfície do terreno. 0 resultado final é uma imagem de alta resolução que permite identificar as diversas interfaces presentes no local.

Os dados GPR foram adquiridos na forma de perfis de reflexão com afastamento constante e na forma de sondagens de velocidade do tipo Wide Angle Reflection and Refraction - WARR. A Figura 3 mostra 


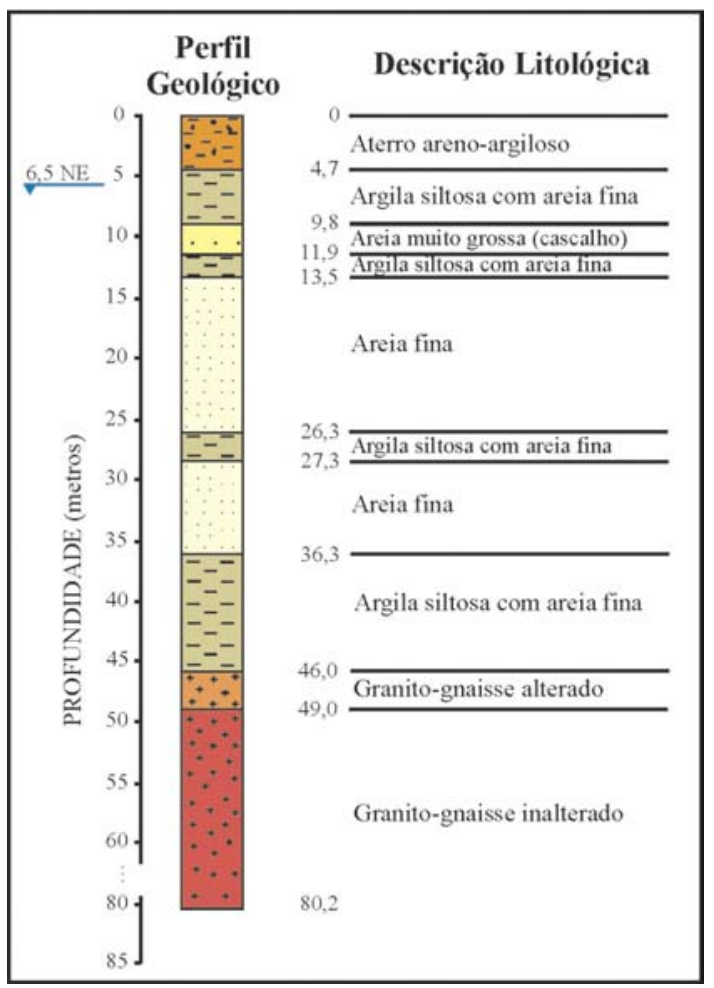

Figura 2 - Perfil litológico do poço (PORSANI, 2001). Figure 2 - Lithologic profile from borehole (PORSANI, 2001).

um perfil de reflexão de $216 \mathrm{~m}$ de comprimento, obtido com antenas de 50MHz. 0 espaçamento entre cada traço foi de $0,5 \mathrm{~m}$ e 0 stack de 512 . As sondagens de velocidade adquiridas com a técnica WARR permitiram calcular a velocidade de $0,08 \mathrm{~m} / \mathrm{ns}$, importante para a conversão do perfil de reflexão em tempo para profundidade.

Na aquisição dos dados utilizou-se o equipamento Sueco Ramac (Mala-GeoScience) pertencente ao Departamento de Geofísica do IAG/ USP, e os dados foram processados utilizando-se 0 software Gradix da Interpex Ltd., USA.

0 método da eletrorresistividade é provavelmente um dos mais utilizados mundialmente, principalmente através da técnica de sondagem elétrica vertical. Neste método as técnicas de aquisição de dados mais usuais são: Sondagem Elétrica Vertical - SEV e Caminhamento Elétrico - CE. Além disso, existe uma variedade de arranjos de eletrodos, por exemplo, Schlumberger, Wenner, dipólo-dipólo, etc., conferindo ao método uma enorme versatilidade (ELIS, 1999).
Nesta pesquisa, utilizamos as técnicas de SEV e CE. As SEV's foram adquiridas utilizando-se 0 arranjo Schlumberger e foram denominadas SEV01-IAG/FIS e SEV02-IAG/FIS. A primeira teve oS eletrodos de emissão de corrente $(A B / 2)$ distanciados até $400 \mathrm{~m}$, alinhados paralelamente à rua do Matão na posição 165m da Linha IAG/FIS. A SEV02-IAG/FIS teve os eletrodos de emissão de corrente ( $A B / 2)$ distanciados até $200 \mathrm{~m}$, alinhados perpendicularmente à linha IAG/FIS na posição 200m. A SEV02-IAG/FIS foi realizada perpendicularmente à rua do Matão devido a suspeita da presença de uma zona de falha paralela e próxima a Linha IAG/FIS.

Os dados de SEV foram modelados através do algoritmo de inversão ID utilizando-se o software Resixip da Interpex Ltd. USA. A Figura 3 mostra os dados da SEV01-IAG/FIS, o modelo geolétrico obtido da inversão 1D e o modelo geológico correspondente.

Foram adquiridos dois perfis de CE com arranjo do tipo dipólodipólo: um com o espaçamento dos eletrodos de $10 \mathrm{~m}$ (CE-10) e outro 
Inst. Física

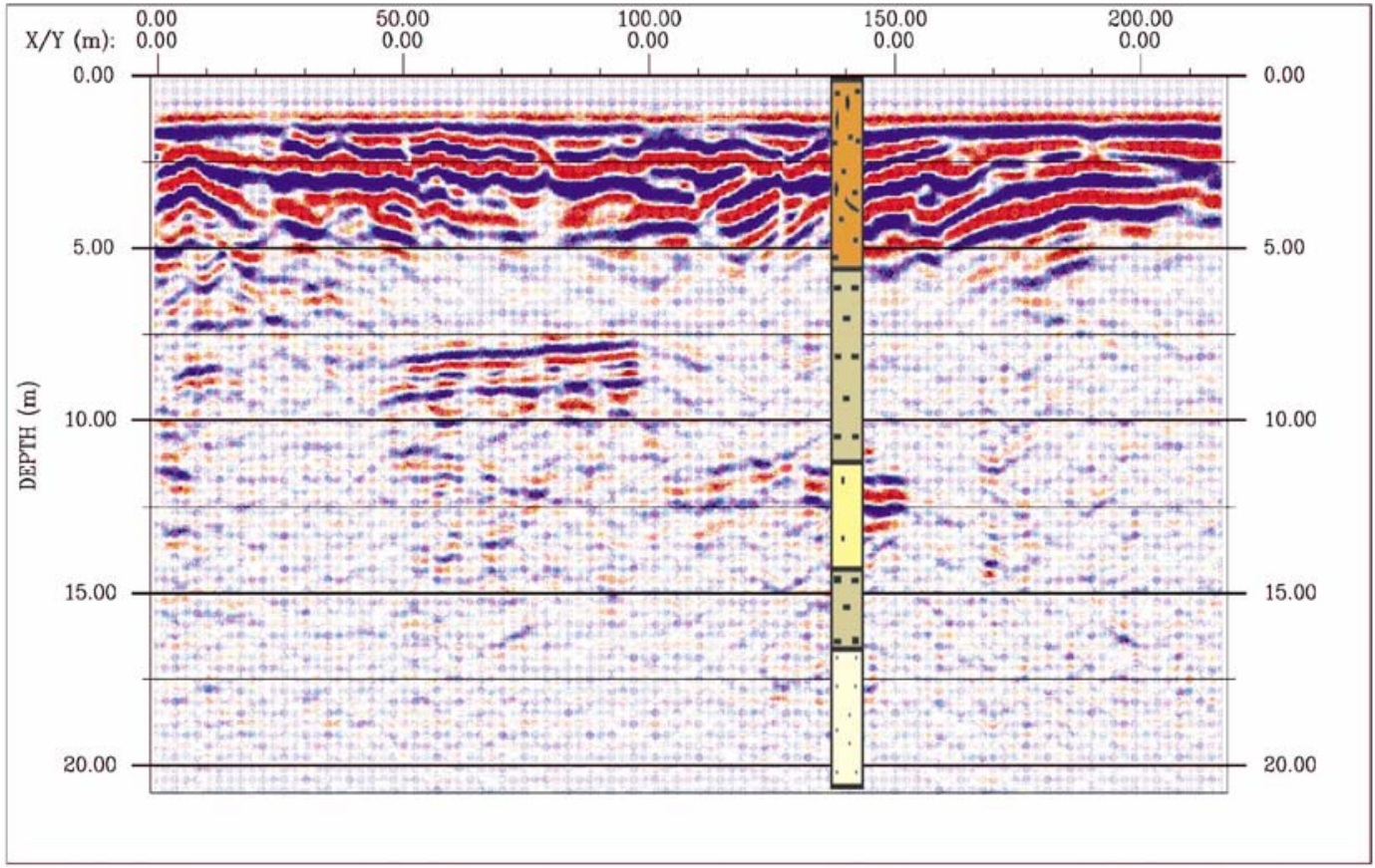

Figura 3 - Perfil de reflexão GPR PERF01-IAG (50MHz) realizado em frente ao IAG/USP, com correspondência litológica do poço PI sobreposto. Figure 3- GPR profile PERFOI-IAG (50 MHz) acquired in front of the IAG/USP and correlation with lito-stratigraphy of PI borehole.

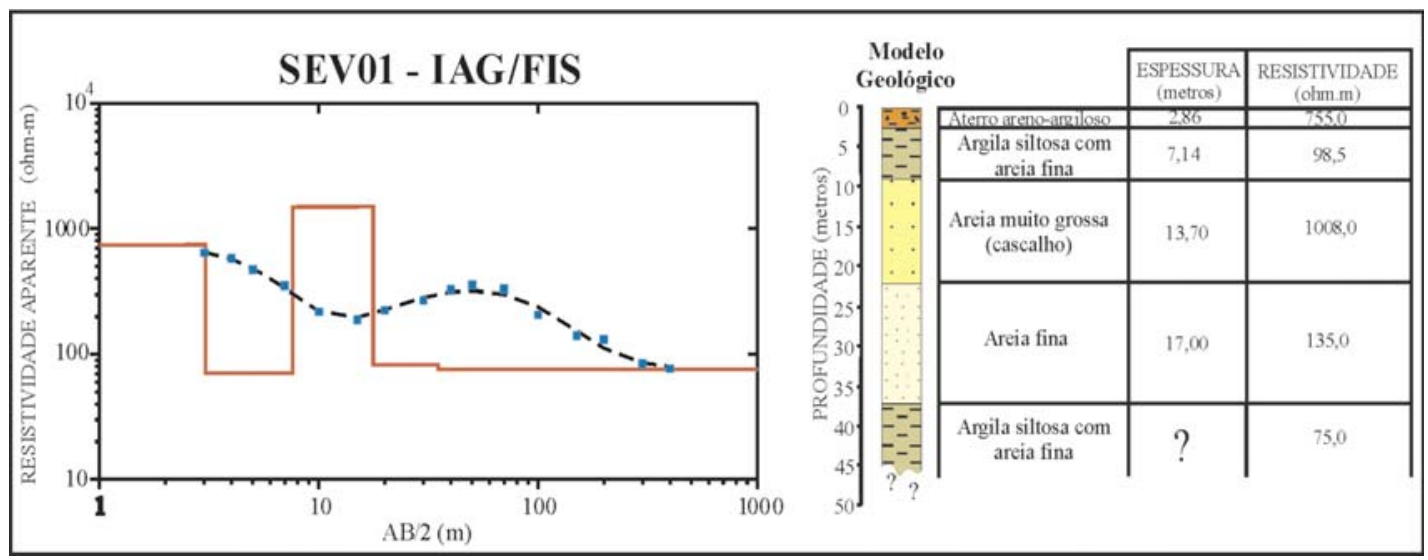

Figura 4 - SEVOI - IAG/FIS. a) Os dados são representados por $\square$ e a linha pontilhada é o resultado do ajuste do modelo de Terra $1 D$. A linha cheia é o modelo de Terra ID ajustada aos dados. Erro de conversão de 7,74\%. b) Modelo geológico-geoelétrico para a SEVO1 - IAG/FIS, proposto com base no perfil litológico do poço.

Figure 4-SEVOI-IAG/FIS. a) The data are represented by $\square$ and dotted line is resulted by fit of ID Earth model. The full line is 10 Earth model fitted by data. The error of data conversion is about 7.7\%. b) Geological-Geophysical model from SEVOI-IAG/FIS, adjust from borehole lithologic profile. 
com espaçamento de 20m (CE-20). Ambos os caminhamentos foram realizados sobre a linha IAG/FIS e tiveram cinco níveis de investigação, atingindo a profundidade teórica de 30m (CE-10) e 60m (CE-20).

Os dados do CE foram apresentados utilizando o soffware Surfer 7.0 da Golden Soffware, Inc. Neste trabalho apresentaremos apenas 0 resultado do CE-20 (Figura 5).

\section{DISCUSSÃO DOS RESULTADOS}

0 perfil de radar PERFO1-IAG, de 50MHz tem $216 \mathrm{~m}$ de comprimento (Figura 3) é caracterizado por vários horizontes refletores irregulares até cerca de $5 \mathrm{~m}$, além da presença de mais dois refletores. A base dos refletores é interpretada como o limite entre o solo/aterro e os sedimentos da Bacia de São Paulo. 0 primeiro refletor, localizado entre 50 e $110 \mathrm{~m}$ no perfil, encontra-se a $8 \mathrm{~m}$ de profundidade com um leve mergulho em direção a NE sendo relacionado à interferência da parede do prédio do Instituto de Física. Esta interpretação foi realizada após as análises dos perfis GPR perpendiculares ao PERF01-IAG e das sondagens de velocidade WARR. Sabe-se que refletores horizontais provocados por objetos superficiais variam de inclinação de acordo com a orientação dos perfis GPR.

0 segundo refletor subhorizontal que ocorre a $12 \mathrm{~m}$ de profundidade, entre as distâncias $100 \mathrm{e} 160 \mathrm{~m}$ foi correlacionado, com base no perfil geológico do poço $\mathrm{PI}$, ao topo de uma camada de areia muito grossa (cascalho). A partir deste refletor não se observa nenhum refletor que mereça destaque. Este fato é atribuído à elevada condutividade dos sedimentos que absorvem e atenuam a propagação da onda eletromagnética do GPR.

A análise quantitativa dos dados de SEV's permitiu caracterizar os materiais de subsuperfície, determinando-se as espessuras e as resistividades das camadas (Figura 4). Nota-se que 0 topo da camada resistiva, observado em torno de $10 \mathrm{~m}$ de profundidade, coincide com 0 refletor horizontal visto no perfil GPR (Figura 3) e está relacionado com uma camada de areia grossa, conforme mostra o perfil litológico do poço Pl (Figura 2). A SEV02 apresentou o mesmo comportamento da SEV01, não sendo apresentada neste trabalho.

No perfil de caminhamento elétrico CE-20 (Figura 5) é nítida a presença de duas regiões resistivas, descontínuas entre $80 \mathrm{~m}$ e $110 \mathrm{~m}$. Nesta região, tem-se uma zona condutora verticalizada, que provavelmente está relacionada com a presença de uma zona de falhas. 0 horizonte resistor que ocorre entre 120 e 190m é subhorizontalizado, e está relacionado com o refletor identificado pelo GPR (Figura 3) e correlacionado com uma camada de areia grossa observada no poço PI (Figura 2).

\section{CONCLUSÕES PRELIMINARES}

As camadas argilosas das formações São Paulo e Itaquaquecetuba atenuam a propagação das ondas eletromagnéticas do radar e são excelentes condutoras de corrente elétrica. As SEV's e caminhamentos não conseguiram atingir 0 embasamento da bacia na região devido à presença destas camadas argilosas e também por falta de espaço físico

\section{Pseudo-Seção de Resistividade Aparente (Ohm.m)}

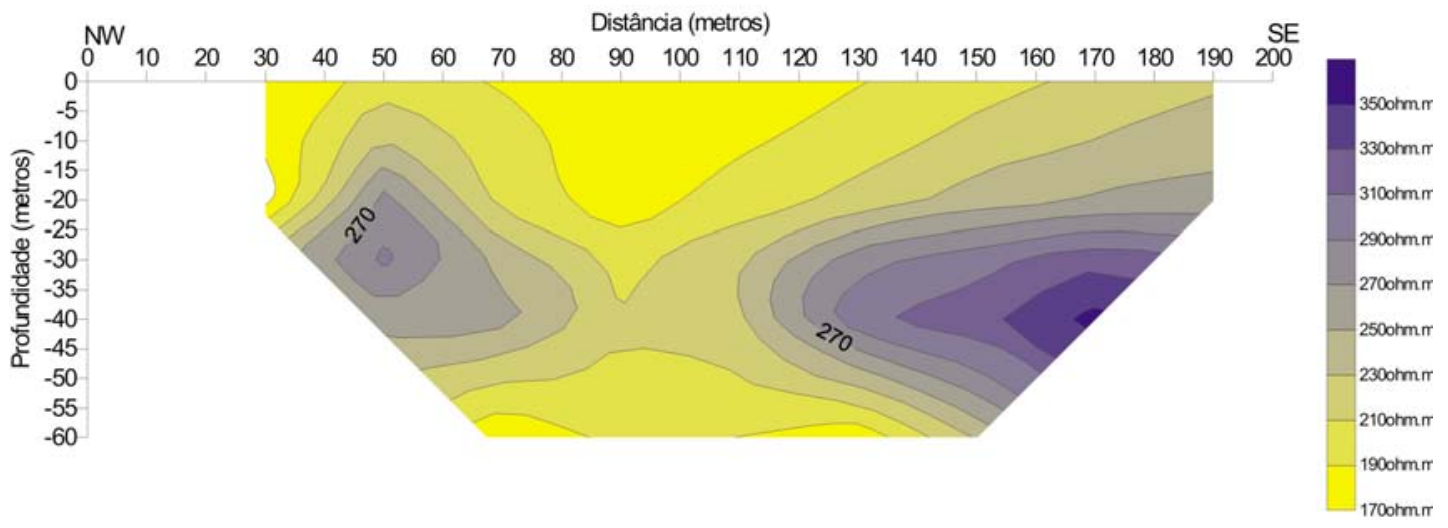

Figura 5 - Pseudo-Seção de Resistividade Aparente do Caminhamento Elétrico CE-20, IAG/FIS.

Figure 5-Pseudo-section of apparent resistivity from CE-20 electrical sounding. 
para ampliar 0 arranjo dos eletrodos. A presença de uma zona condutora entre $80 \mathrm{~m}$ e $110 \mathrm{~m}$, vista no CE-20, é um indicativo da presença de uma zona de falhas, devido a mudança lateral brusca de condutividade elétrica. Esta hipótese ainda será checada, mas parece haver uma correspondência com a variação da feição topográfica observada no campo.

0 método GPR mostra-se bastante promissor para investigações na Bacia de São Paulo até a profundidade de $20 \mathrm{~m}$, delineando com clareza as camadas de sedimentos grossos, bem como a espessura do solo/aterro. Este resultado está de acordo com as informações obtidas do poço Pl.

\section{Agradecimentos}

A Fapesp, pela concessão da bolsa de mestrado (processo 99/ 12217-5) e ao Departamento de Geofísica do IAG pela oportunidade de realizar esta pesquisa. Aos professores doutores Jorge Luís Porsani, pela orientação e Vagner Roberto Elis, pelas discussões e ajuda na aquisição de dados.

\section{REFERÊNCIAS}

ELIS, V. R. Avaliação da aplicabilidade de métodos elétricos de prospecção geofísica no estudo de áreas utilizadas para disposição de resíduos. 1999. 264 p. Tese (Doutorado)-Instituto de Geociências e Ciências Exatas, Universidade Estadual Paulista, Rio Claro, 1999.

IRITANI, M. A. Potencial hidrogeológico da Cidade Universitária de São Paulo. 1993. Dissertação (Mestrado)-Universidade de São Paulo, São Paulo, 1993.

PORSANI, J. L. Ground Penetrating Radar (GPR): proposta metodológica de emprego em estudos geológico-geotécnicos nas regiões de Rio Claro e Descalvado - SP. 1999. 145 p. Tese (Doutorado)-Instituto de Geociências e Ciências Exatas, Universidade Estadual Paulista, Rio Claro, 1999.

- Investigações geofísicas de superfície e de poço na borda da bacia sedimentar de São Paulo. 2001. Relatório Parcial. Projeto Fapesp (99/12215-2). 50p.

\section{NOTAS SOBRE OS AUTORES}

Welitom Rodrigues Borges é geólogo pelo Instituto de Ciências Exatas e da Terra da Universidade Federal de Mato Grosso (ICET/UFMT) em 2000. Em 2002 obteve o título de Mestrado em Ciências, área de Geofísica Aplicada, pelo Instituto de Astronomia, Geofísica e Ciências Atmosféricas da Universidade de São Paulo (IAG/USP). Atualmente está fazendo o Doutorado (bolsista Fapesp 02/07510-0) junto ao Programa de Pós-Graduação em Geofísica do IAG/USP, desenvolvendo a pesquisa "Caracterização geofísica de alvos rasos com aplicações no planejamento urbano, meio ambiente e arqueologia: estudo sobre o sí́io controlado do IAG/USP".

Jorge Luís Porsani é geólogo pelo Instituto de Geociências da UFBa (1987). Mestrado em Geofísica pelo Núcleo de Pesquisas Geofísicas Aplicadas à Prospecção de Hidrocarbonetos da UFPa (1991). De 1991 a 1996, trabalhou como Geofísico no Centro de Pesquisas da PETROBRAS. Doutorado em Geociências e Meio Ambiente pelo Instituto de Geociências e Ciências Exatas da UNESP (1999). Desde 01/12/ 1998 é Docente do Departamento de Geofísica do IAG/USP, atuando com métodos geoelétricos aplicados à geologia, geotecnia, meio ambiente e arqueologia. Atualmente, é vice-coordenador do Programa de Pós-Graduação em Geofíisica e coordenador do Projeto de Instalação do Sítio Controlado de Geofísica Rasa do IAG (Fapesp 02/07509-1). 